\title{
A Compositional Symbolic Verification Framework for Concurrent Software
}

\author{
Conghua Zhou \\ School of Computer Science and Telecommunication Engineering, Jiangsu University \\ No. 301 Xufu Road \\ Zhenjiang, China, 212013 \\ chzhou@mail.edu.cn
}

\begin{abstract}
For concurrent software systems state/event linear temporal logic SE-LTL is a specification language with high expressive power and the ability to reason about both states and events. Until now, SE-LTL model checking algorithm is still explicit. For SE-LTL we provide a SAT-based Bounded Model Checking procedure. We also present a framework for model checking concurrent software systems which integrates three powerful verification techniques, SAT-based Bounded Model Checking, counterexample-guided abstraction refinement and compositional reasoning. In the framework the abstraction and refinement steps are performed over each component separately, and the model checking step is symbolic.
\end{abstract}

\section{Categories and Subject Descriptors}

D.2.4 [Software Engineering]: Software/Program Verification - Model checking, Formal methods

\section{General Terms}

Verification

\section{Keywords}

model checking, SAT, abstract, composition

\section{INTRODUCTION}

The specification logic SE-LTL[3] is a state/event derivative of LTL[5]. This allows us to represent both software implementations and specifications directly without any program annotations or privileged insights into program execution. Chaki further showed how standard automatatheoretic LTL model checking algorithms can be ported to their framework at no extra cost, enabling them to directly

\footnotetext{
*Supported by the National Natural Science Foundation of China No.60603041, the Province Natural Science Foundation of Jiangsu No.BK2006073
}

Permission to make digital or hard copies of all or part of this work for personal or classroom use is granted without fee provided that copies are not made or distributed for profit or commercial advantage and that copies bear this notice and the full citation on the first page. To copy otherwise, or republish, to post on servers or to redistribute to lists, requires prior specific permission and/or a fee.

INFOSCALE 2007, June 6-8, Suzhou, China

Copyright $@ 2007$ ICST 978-1-59593-757-5

DOI 10.4108/infoscale.2007.906 benefit from the large body of research on efficient LTL verification. However, until now SE-LTL model checking algorithm is still explicit.

Bounded Model Checking(BMC)[2] based on SAT methods has recently been introduced as a complementary technique to BDD-based symbolic model checking[6]. In this paper for SE-LTL we provid its SAT-based BMC procedure. Given an LKS $M$, an SE-LTL formula $\phi$ and a natural number $k$, our BMC procedure decides whether there exists a computation in $M$ of length $k$ or less that violates $\phi$, i.e. $M \models_{k} E \neg \phi$. Our BMC is performed by generating a propositional formula, which is satisfiable if and only if such a computation exists.

We also present an efficient verification strategy which combines SAT-based BMC, counterexample-guided abstraction refinement $[1,4]$ and compositional reasoning[7]: starting with a coarse initial abstraction, our scheme computes increasingly precise abstractions of the target system by analyzing spurious counterexamples until either a real counterexample is obtained or the system is found to be correct.

More precisely, given a system $M$ composed of $n$ concurrent components $M_{1}, \ldots, M_{n}$, and a SE-LTL specification $\phi$, the verification of $M=\phi$ proceeds as follows:

(1) Abstract. Create an abstraction $A$ such that all behaviors of $A$ are preserved by $M$. This is done componentwise without constructing the full state space of $M$.

(2) Verify. Verify whether $\left.A\right|_{k} E \neg \phi$. If so, extract an abstract counterexample $C$ that indicates in which $\phi$ fails in $A$. Otherwise check whether $k \leq C T$. If so, let $k=k+1$, goto 2 . Otherwise return $M \models \phi$.

(3) Check whether $C$ gives rise to a real counterexample over $A$. If $C$ corresponds to a genuine behavior of $M$ then report a failure along with a fragment of each $M_{i}$ that illustrates why $M \not \models \phi$. If $C$ is spurious, on the other hand, refine $A$ using $C$ to obtain a more precise abstraction and repeat from step 1 . This refinement step, like the initial abstraction, is performed component-wise.

Of the three steps in this abstract-verify-refine process only the verification stage of our technique requires the explicit composition of a system. The other stages can be performed one component at a time. To the best of our knowledge, our strategy is the first SAT-based, counterexampleguided, compositional abstraction refinement scheme to perform verification of linear time temporal specifications. 


\section{PRELIMINARIES}

\subsection{Labeled Kripke Structure}

Definition 1. A labeled Kripke structure $M$ is a 6-tuple $\left(S, s_{0}, A P, \Sigma, L, R\right)$ where, $S$ is a finite non-empty set of states; $s_{0} \in S$ is an initial state; $A P$ is a finite set of atomic state propositions; $\Sigma$ is a finite set of events; $L: S \rightarrow 2^{A P}$ is a state-labeling function; $R \subseteq S \times \Sigma \times S$ is a transition relation that must be total, that is, for every state $s \in S$ there is an event $a \in \Sigma$ and a state $s^{\prime} \in S$ such that $R\left(s, a, s^{\prime}\right)$ holds.

Given an LKS $M=\left(S, s_{0}, A P, \Sigma, L, R\right)$ we write $S(M)$, $s_{0}(M), A P(M), L(M), \Sigma(M)$ and $R(M)$ to mean $S, s_{0}$, $A P, L, \Sigma$ and $R$ respectively. A path $\pi=s_{0}, a_{0}, s_{1}, a_{1}, \ldots$ of $M$ is an alternating infinite sequence of states and events subject to the following: for each $i \geq 0, s_{i} \in S, a_{i} \in \Sigma$ and $R\left(s_{i}, a_{i}, s_{i+1}\right)$ holds. For the path $\pi=s_{0}, a_{0}, s_{1}, a_{1}, \ldots$ we use $\pi(i)$ to denote the $i$ th state $s_{i}$, use $\pi(i, E)$ to denote the $i$ th event $a_{i}$. We right Path $(M)$ to denote the set of infinite and finite paths whose first state is $s_{0}(M)$.

Definition 2. A path $\pi$ is a $(k, l)$-loop, with $l<k$, if $(\pi(k), \pi(k, E), \pi(l)) \in R$ and $\pi=u \cdot v^{\omega}$, where $u=\pi(0)$, $\pi(0, E), \ldots, \pi(l-1), \pi(l-1, E)$ and $v=\pi(l), \pi(l, E), \ldots, \pi(k)$, $\pi(k, E)$. We call $\pi$ simply a $k$-loop if there is an integer $l$ with $0 \leq l \leq k$ such that which $\pi$ is a $(k, l)$-loop.

\subsection{Abstraction}

The notion of abstraction is central to our approach.We list below the properties that we require of any abstraction scheme to be usable in our framework. Let $M=$ $\left(S, s_{0}, A P, \Sigma, L, R\right)$ and $A=\left(S^{A}, s_{0}^{A}, A P^{A}, \Sigma^{A}, L^{A}, R^{A}\right)$ be two LKSs. We say that $A$ is an abstraction of $M$, written $M \subseteq A$ iff $A P^{A} \subseteq A P, \Sigma^{A}=\Sigma$, for every path $\pi=s_{0}, a_{0}, s_{1}, a_{1}, \ldots$ of $M$ there exists a path $\pi^{\prime}=s_{0}^{\prime}$, $a_{0}^{\prime}, s_{1}^{\prime}, a_{1}^{\prime}, \ldots$ of $A$ such that for each $i \geq 0, a_{i}^{\prime}=a_{i}$ and $L^{A}\left(s_{i}^{\prime}\right)=L\left(s_{i}\right) \cap A P^{A}$.

\subsection{Parallel Composition}

Let $M_{1}=\left(S_{1}, s_{0}^{1}, A P_{1}, \Sigma_{1}, L_{1}, R_{1}\right)$ and $M_{2}=\left(S_{2}, s_{0}^{2}\right.$, $\left.A P_{2}, \Sigma_{2}, L_{2}, R_{2}\right)$ be two LKSs. $M_{1}$ and $M_{2}$ are said to be compatible, i.e., that they do not share variables: $S_{1} \cap S_{2}=$ $A P_{1} \cap A P_{2}=\emptyset$. The parallel composition of $M_{1}$ and $M_{2}$ is given by $M_{1} \| M_{2}=\left(S_{1} \times S_{2}, s_{0}^{1} \times s_{0}^{2}, A P_{1} \cup A P_{2}, \Sigma_{1} \cup\right.$ $\left.\Sigma_{2}, L_{1} \cup L_{2}, R\right)$ where $\left(L_{1} \cup L_{2}\right)\left(s_{1}, s_{2}\right)=L_{1}\left(s_{1}\right) \cup L_{2}\left(s_{2}\right)$ and $R\left(\left(s_{1}, s_{2}\right), a,\left(s_{1}^{\prime}, s_{2}^{\prime}\right)\right)$ holds iff one of the following holds:

1. $a \in \Sigma_{1} \backslash \Sigma_{2}$ and $R_{1}\left(s_{1}, a, s_{1}^{\prime}\right)$ holds and $s_{2}^{\prime}=s_{2}$.

2. $a \in \Sigma_{2} \backslash \Sigma_{1}$ and $R_{2}\left(s_{2}, a, s_{2}^{\prime}\right)$ holds and $s_{1}^{\prime}=s_{1}$.

3. $a \in \Sigma_{1} \cap \Sigma_{2}$ and $R_{1}\left(s_{1}, a, s_{1}^{\prime}\right)$ holds and $R_{2}\left(s_{2}, a, s_{2}^{\prime}\right)$ holds.

Let $M_{1}$ and $M_{2}$ be as above, and let $\pi=\left(s_{0}^{1}, s_{0}^{2}\right), a_{0}, \ldots$ be an alternating infinite sequence of states and events of $M_{1} \| M_{2}$. The projection $\pi \uparrow M_{i}$ of $\pi$ on $M_{i}$ consists of(possibly finite) the subsequence of $s_{0}^{i}, a_{0}, \ldots$ obtained by simply removing all pairs $\left(a_{j}, s_{j+1}^{i}\right)$ for which $a_{j} \notin \Sigma_{i}$. In other words, we keep from $\pi$ only those states that belong to $M_{i}$, and excise any transition labeled with an event not in $\Sigma_{i}$.

\subsection{State/Event Linear Temporal Logic SE-LTL}

Given an LKS $M=\left(S, s_{0}, A P, \Sigma, L, R\right)$, we consider linear temporal logic state/event formulas SE-LTL over the sets $A P, \Sigma$ as follows:

- if $p \in A P$ then $p, \neg p$ is an SE-LTL formula; if $\sigma \in \Sigma$ then $\sigma$ is an SE-LTL formula.

- if $f$ is an SE-LTL formula, then $X f, F f, G f$ are SELTL formulas.

- if $f, g$ are SE-LTL formulas, then $f \wedge g, f \vee g, f U g, f R g$ are SE-LTL formulas.

Definition 3. (Semantics of SE-LTL) Let $\pi=s_{0}, a_{0}, \ldots$ be an infinite path, $\pi^{i}$ stands for the suffix of $\pi$ starting in state $s_{i} . \pi \mid=f$ is inductively defined as follows:

- $\pi \models p$ if and only if $p \in L(\pi(0))$.

- $\pi \models \neg p$ if and only if $p \notin L(\pi(0))$.

- $\pi \models a$ if and only if $\pi(0, E)=a$.

- $\pi \models f \wedge g$ if and only if $\pi \models f$ and $\pi \models g$.

- $\pi \models f \vee g$ if and only if $\pi \models f$ or $\pi \models g$.

- $\pi \models X f$ if and only if $\pi^{1} \models f$.

- $\pi \models F f$ if and only if for some $i \geq 0, \pi^{i} \models f$.

- $\pi \models G f$ if and only if for all $i \geq 0, \pi^{i} \models f$.

- $\pi \models f U g$ if and only if there is some $i \geq 0$ such that $\pi^{i} \models g$ and for all $j \leq i-1, \pi^{j} \models f$.

- $\pi \models f R g$ if and only if for all $i \geq 0, \pi^{i} \models g$ or there is some $i \geq 0$ such that $\pi^{i} \models f$ and for all $j \leq i, \pi^{j} \models g$.

Definition 4. $M \models f$ if and only if for all path $\pi$ of $M$, $\pi \models f . M \models E f$ if and only if there is a path $\pi$ of $M$ such that $\pi \models f$.

\section{SAT-BASED BOUNDED MODEL CHECK- ING FOR SE-LTL}

Bounded model checking based on SAT methods has been introduced as a complementary technique to BDD-based symbolic model checking. The main idea of bounded model checking is to search for an execution of the system of some length $k$, which constitutes a counterexample for a verified property. To perform bounded model checking on SE-LTL, we first define a bounded semantics for SE-LTL, which is an approximation to the unbounded semantics. Second we reduce bounded model checking to propositional satisfiability. Third we discuss the completeness threshold for bounded model checking on SE-LTL.

\subsection{Bounded Semantics for SE-LTL}

In bounded model checking a crucial observation is that the prefix of a path is finite, it still might represent an infinite path if there is a back loop from the last state of the prefix to any of the previous states. If there is no such back loop, then the prefix does not say anything about the infinite behavior of the path. Thus when we define bounded semantics for SE-LTL we must consider whether a finite path represents an infinite behavior. 
Definition 5. (Bounded Semantics for a Loop) Let $\pi$ be a $k$-loop. Then an SE-LTL formula $f$ is valid along the path $\pi$ with bound $k$ (in symbols $\pi=_{k} f$ ) iff $\pi \models f$.

Definition 6. (Bounded Semantics without a Loop) Let $\pi$ be a path that is not k-loop. Then an SE-LTL formula $f$ is valid along $\pi$ with bound $k$ (in symbols $\pi \models_{k} f$ iff $\pi \models{ }_{k}^{0} f$ where

- $\pi \mid=_{k}^{i} p$ iff $p \in L(\pi(i)) ; \pi \models_{k}^{i} \neg p$ iff $p \notin L(\pi(i))$.

- $\pi=_{k}^{i} a$ iff $a \equiv \pi(i, E)$.

- For all other cases, refer to [2].

We use the notation $M \models_{k} E f$ to represent that there exists a path $\pi$ of $M$ such that $\pi \models_{k} f$.

Theorem 1. Let $A P$ be a set of propositions, $M$ be an LKS over $A P, \pi$ be a path of $M, f$ be an SE-LTL formula, and $k$ be a bound. Then $\pi=_{k} f$ implies $\pi \models f$.

If $\pi$ is a $k$-loop then the conclusion in Theorem 1 follows by the definition. In the other case we can prove the conclusion by induction over the structure of $f$ straightly.

Definition 7. For every LKS $M$ and an SE-LTL property $f$, the natural number $k$ called a $C T$ of $f$ if and only if the following condition holds: if there is no counterexample to $f$ in $M$ of length $k$ or less, then $M \models f$.

Theorem 2. Let $A P$ be a set of propositions, $M$ be an LKS over $A P, \pi$ be a path of $M, f$ be an SE-LTL formula, and $k$ be a natural number. Then $M \models E f$ implies there exists a bound $k \leq|M| \times 2^{|f|}$ such that $M=_{k} E f$. In other words, $|M| \times 2^{|f|}$ is a $C T$ of $f$.

\subsection{Translation}

In the previous section we defined the semantics for bounded model checking. We now show how to reduce bounded model checking to propositional satisfiability. This reduction enables us to use efficient propositional SAT solvers to perform model checking.

Given an LKS $M$, an SE-LTL formula $f$ and a bound $k$, we will construct a propositional formula $[M, f]_{k}$. Let $s_{0}, a_{0}, \ldots, s_{k}, a_{k}$ be a finite sequences of states and events on a path $\pi$. Each $s_{i}$ represents a state at time step $i$ and consists of an assignment of truth values to the set of state variables. Each $a_{i}$ represents an event at time step $i$ and consists of an assignment of truth values to the set of event variables. The formula $[M, f]_{k}$ encodes constraints on $s_{0}, a_{0}, \ldots, s_{k}, a_{k}$ such that $[M, f]_{k}$ is satisfiable iff $\pi$ is a witness for $f$. The definition of formula $[M, f]_{k}$ will be presented as three separate components. We first define a propositional formula $[M]_{k}$ that constraints $s_{0}, a_{0}, \ldots, s_{k}, a_{k}$ to be a valid path starting from the initial state. We then define the loop condition, which is a propositional formula that is evaluated to true only if the path $\pi$ contains a loop. Finally, we define a propositional formula that constrains $\pi$ to satisfy $f$.

Definition 8. (Unfolding the Transition Relation) For the bound $k$, we define $[M]_{k}:=I\left(s_{0}\right) \wedge \bigwedge_{j=0}^{k-1} R\left(s_{i}, a_{i}, s_{i+1}\right)$, where $I\left(s_{0}\right)$ is true if and only if $s_{0}$ is the initial state.
The translation of an SE-LTL formula depends on the shape of the path $\pi$. We define the propositional formula ${ }_{l} L_{k}$ to be true if and only if there is a transition from sate $s_{k}$ to state $s_{l}$.

Definition 9. (Loop condition) For two integers $k, l$ with $k \geq l \geq 0$, let ${ }_{l} L_{k}:=R\left(s_{k}, a_{k}, s_{l}\right)$.

Depending on whether a path is a $k$-loop, we have two different translations of a SE-LTL formula $f$. First we consider the case where the path is a $k$-loop. We give a recursive translation of an SE-LTL formula $f$ for a $k$-loop path $\pi$. The translation of $f$ recurses over its subterms and the states in $\pi$. The intermediate formula $l_{l}[]_{k}^{i}$ depends on three parameters: $l, k$ and $i$. We use $l$ for the start position of the loop, $k$ for the bound, and $i$ for the current position in $\pi$.

Definition 10. (Translation of an SE-LTL formula on a $(k, l)$-loop $)$

- ${ }_{l}[a]_{k}^{i}:=\left(a \equiv a_{i}\right)$;

- For all other cases, refer to [2].

For the case where $\pi$ is not a $k$-loop, the translation can be treated as a special case of the $k$-loop translation. For LKS with total transition relations, every finite path $\pi$ can be extended to an infinite one. Since the property of the path beyond event $a_{k}$ is unknown, we make a conservative approximation and assume all properties beyond $a_{k}$ are false.

Definition 11. (Translation of an SE-LTL formula without a Loop)

- $[a]_{k}^{i}:=\left(a \equiv a_{i}\right)$

- For all other cases, refer to [2].

Combining all components, the encoding of a bounded model checking problem in propositional logic is defined as follows.

Definition 12. (General translation) Let $M$ be an LKS, $f$ be an SE-LTL formula, and $k$ be a bound. We define $[f]_{k}:=\left([f]_{k}^{0} \vee \bigvee_{l=0}^{k}\left({ }_{l} L_{k} \wedge_{l}[f]_{k}^{0}\right)\right.$ and $[M, f]_{k}:=[M]_{k} \wedge[f]_{k}$

The translation scheme guarantees the following theorem, which we state without proof.

Theorem 3. Let $M$ be an LKS, $f$ be an SE-LTL formula, and $k$ be a bound. Then $[M, f]_{k}$ is satisfiable if and only if $M \models{ }_{k} E f$.

Thus, the reduction of bounded model checking to SAT is sound and complete with respect to the bounded semantics.

Corollary 1. Let $M$ be an LKS, $f$ be an SE-LTL formula, and $k$ be a bound. $M=E f$ if and only if there exists an integer $k \leq|M| \times 2^{|f|}$ such that $[M, f]_{k}$ is satisfiable.

\section{COMPOSITIONAL SAT-BASED SE-LTL VERIFICATION}

We now discuss how our framework enables us to verify SE-LTL specification on parallel compositions of LKSs incrementally and compositionally. When trying to determine whether an SE-LTL specification holds on a given LKS, the following result is the key ingredient needed to exploit abstractions in the verification process. 
Theorem 4. Let $M$ and $A$ be LKSs with $M \subseteq A$. Then for any SE-LTL formula $\phi$ over $M$ which mentions only propositions (and events) of $\mathrm{A}$, if $A \models \phi$ then $M \models \phi$.

Suppose now that we are given a collection $M_{1}, \ldots, M_{n}$ of LKSs, as well as an SE-LTL specification $\phi$, with the task of determining whether $M_{1}\|\ldots\| M_{n} \models \phi$. We first create initial abstractions $M_{1} \subseteq A_{1}, \ldots, M_{n} \subseteq A_{n}$. Then we check whether $A_{1}\|\ldots\| A_{n} \models_{k} \neg \phi$. In the affirmative, we are provided with an abstract counterexample $\pi$ such that $\pi \models_{k} \neg \phi$. We must then determine whether this counterexample is real or spurious, i.e., whether it corresponds to a counterexample in $M_{1}\|\ldots\| M_{n}$ or not. In the negative, we check whether $k \leq C T$. If so, then check whether $A_{1}\|\ldots\| A_{n} \models_{k+1} \neg \phi$. Otherwise return that $M_{1}\|\ldots\| M_{n} \models$ $\phi$.

This counterexample validation check can be performed compositionally, as follows. The counterexample is real iff for each $i$, the projection $\pi \uparrow A_{i}$ corresponds to (the prefix of) a valid behavior of $M_{i}$. To this end, we 'simulate' $\pi \uparrow A_{i}$ on $M_{i}$. If $M_{i}$ accepts the path, we go on to the next component. Otherwise, we refine our abstraction $A_{i}$, yielding a new abstraction $A_{i}^{\prime}$ with $M_{i} \subseteq A_{i}^{\prime} \subseteq A_{i}$ and such that $A_{i}^{\prime}$ also rejects the projection $\pi \uparrow A_{i}^{\prime}$ of the spurious counterexample $\pi$.

Let us return to our SE-LTL specification $\phi$, and let us fix throughout $P_{\phi}$ to be the set of all atomic state propositions appearing in $\phi$. Consider any of the $M_{i}^{\prime}$ s. An abstraction of $M_{i}$ is entirely determined by a $P_{\phi}$-respecting partition $\approx_{i}$ of the set of states of $M_{i}$ : such an abstraction is denoted $M_{i} / \approx_{i}$.

The initial abstraction $M_{i} / \approx_{i}^{1}$ is the coarsest possible: $s \approx_{i}^{1}$ iff $L\left(M_{i}\right)(s) \cap P_{\phi}=L\left(M_{i}\right)\left(s^{\prime}\right) \cap P_{\phi}$. Suppose now that we are handed $\pi_{i} \in \operatorname{Path}\left(M_{i} / \approx_{i}^{k}\right)$ (i.e., $\pi_{i}$ is either a lasso or a finite path of $M_{i} / \approx_{i}^{k}$. We must determine whether $\pi_{i}$ is a real or spurious counterexample component, i.e., whether $\pi_{i}$ gives rise to a valid path of $M_{i}$ or not. Moreover, in the latter case, we want to refine our partition $\approx_{i}^{k}$ into $\approx_{i}^{k+1}$ so that $\pi_{i}$ is rejected by $M_{i} / \approx_{i}^{k+1}$.

The validation/refinement step is similar to that originally proposed by S. Chaki et al.[3]. Our validation/refinement step proceeds as follows. For any set $Q$ of states of $M_{i}$ and event $a$, let $\operatorname{Succ}(Q, a)=\left\{s^{\prime} \mid \exists s \in Q,\left(s, a, s^{\prime}\right) \in R\right\}$ denote the set of $a$-successors of $Q$ in $M_{i}$. Let us first suppose that $\pi_{i}=s_{0}, a_{0}, s_{1}, a_{1}, \ldots, a_{m-1}, s_{m}, a_{m}$ is a finite path of $M_{i} / \approx_{i}^{k}$. Start with the set $Q_{0}=\left\{s_{0}\left(M_{i}\right)\right\} \cap s_{0}$ and compute successively $Q_{j+1}=\operatorname{succ}\left(Q_{j}, a_{j}\right) \cap s_{j}$. If, upon reaching the end of $\pi, Q_{m+1}$ is non-empty, then clearly $\pi$ is a valid finite path of $M_{i}$. Otherwise, let $Q_{j+1}$ be the first empty set thus generated. Refine the partition $\approx_{i}^{k}$ by splitting $s_{j}$ into $Q_{j}$ and $s_{j}-Q_{j}$, yielding a new partition $\approx_{i}^{k+1}$. It is then easy to see that $M_{i} / \approx_{i}^{k+1}$ will reject $\pi_{i}$.

In case $\pi$ is a lasso, things are slightly more complicated. If $M_{i}$ rejects $\pi$, then the algorithm above will establish his in the very same manner, by eventually producing an empty set of states $Q_{j+1}$. On the other hand, if $\pi$ is accepted by $M_{i}$ then there will be sets of states $Q_{j}=Q_{j+p}$ such that $Q_{j+p}$ is obtained from $Q_{j}$ by following the loop part of $\pi$ a finite number of times. Since all state spaces involved are finite the search will always terminate with one or the other answer after a finite number of iterations.

The full algorithm for checking whether $M_{1}\|\ldots\| M_{n}=\phi$ is given in Fig. 1. Note that the abstraction, counterexamplevalidation, and refinement steps are all performed one com- ponent at a time.

Algorithm SE-LTL Model Checking $\left(M_{1}, \ldots, M_{n} ; \phi\right)$

for $i:=1$ to $n$ : let $A_{i}$ be the initial abstract of $M_{i}$;

for $k:=1$ to $C T$ of $M_{1}\|\ldots\| M_{n}$

$\left\{(1)\right.$ decide whether $\left[A_{1}\|\ldots\| A_{n}, \neg \phi\right]_{k}$ is satisfiable

if $\left[A_{1}\|\ldots\| A_{n}, \neg \phi\right]_{k}$ is satisfiable, then suppose

$\pi$ be path in $A_{1}\|\ldots\| A_{n}$ violating $\phi$;

looking for $i$ such that $\pi \uparrow A_{i}$ is spurious;

if no such $i$ then

return $M_{1}\|\ldots\| M_{n} \not \models \phi$ along with

the counterexample derived from $\pi$;

else refine $A_{i}$, yielding a new abstraction $A_{i}^{\prime}$ with $M_{i} \subseteq A_{i}^{\prime} \subseteq A_{i}$ and $A_{i}^{\prime}$ also rejects $\pi \uparrow A_{i}^{\prime}$;

Let $A_{i}=A_{i}^{\prime}$, goto (1)

else if $\left[A_{1}\|\ldots\| A_{n}, \neg \phi\right]_{k}$ is unsatisfiable

if $k=C T$ then return $M_{1}\|\ldots\| M_{n} \models \phi$

else $k:=k+1$.

Figure 1: The overall SE-LTL model checking algorithm for a concurrent system $M_{1}\|\ldots\| M_{n}$ and specification $\phi$

\section{CONCLUSION AND FUTURE WORK}

In this paper, we presented a SAT-based Bounded Model Checking procedure for the state/event linear time temporal logic SE-LTL. We further provided a new verification strategy for concurrent software system which integrates SATbased BMC, counterexample-guided abstraction refinement and compositional reasoning. We are developing the tool for implementing the new strategy. For future work we would like to discuss other equivalent relations.

\section{REFERENCES}

[1] S. Bensalem, Y. Lakhnech, and S. Owre. Computing abstractions of infinite state systems compositionally and automatically. LNCS, 1254:319-331, 1998.

[2] A. Biere, A. Cimatti, E. M. Clarke, and Y. Zhu. Symbolic model checking without bdds. LNCS, 1579:193-207, 1999.

[3] S. Chaki, E. M. Clarke, J. Quaknine, N. Sharygina, and N. Sinha. Concurrent software verification with states, events, and deadlock. Formal Aspects of Computing, 17(4):461-483, April 2004.

[4] E. M. Clarke, O. Grumberg, S. Jha, Y. Lu, and H. Veith. Counterexample-guided abstraction refinement. LNCS, 1885:154-169, 2000.

[5] E. M. Clarke, O. Grumberg, and D. Peled. Model checking. MIT Press, MA, 1999.

[6] K. L. McMillan. Symbolic model checking. Kluwer Academic Publishers, Netherlands, 1993.

[7] K. L. McMillan. A compositional rule for hardware design refinement. LNCS, 1254:24-35, 1997. 